\title{
Multiple Scanning Effects in Radiochromic Film Dosimetry: A Method to Reduce the Increase of Optical Density
}

\author{
Benjamin Foe Ngono ${ }^{*}$, Alexandre Ngwa Ebongue ${ }^{1,2 *}$, Daniel Bongue1, \\ Albert Mouelle Sone ${ }^{3}$, Moise G. Kwato Njock ${ }^{1}$ \\ ${ }^{1}$ Centre for Atomic Molecular Physics and Quantum Optics (CEPAMOQ), Faculty of Science, University of Douala, Douala, Cameroon \\ ${ }^{2}$ Department of Physics, Faculty of Science, University of Douala, Douala, Cameroon \\ ${ }^{3}$ Department of Radiotherapy and Oncology, Douala General Hospital, Douala, Cameroon \\ Email: *nebalex@yahoo.fr
}

How to cite this paper: Ngono, B.F., Ebongue, A.N., Bongue, D., Sone, A.M. and Njock, M.G.K. (2020) Multiple Scanning Effects in Radiochromic Film Dosimetry: A Method to Reduce the Increase of Optical Density. International Journal of Medical Physics, Clinical Engineering and Radiation Oncology, 9, 34-41.

https://doi.org/10.4236/ijmpcero.2020.91004

Received: January 15, 2020

Accepted: February 23, 2020

Published: February 26, 2020

Copyright $\odot 2020$ by author(s) and Scientific Research Publishing Inc. This work is licensed under the Creative Commons Attribution International License (CC BY 4.0).

http://creativecommons.org/licenses/by/4.0/

\section{c) (i) Open Access}

\begin{abstract}
In this work, a method to reduce increase in optical density (OD) caused by multiple scanning in radiochromic film dosimetry in combination with a flatbed scanner is presented. Gafchromic EBT3 films are scanned with Epson Pro 1680 Expression scanner and time intervals of 15 minutes and 30 minutes are observed between consecutive scans to reduce the increase in temperature of the scanner. The maximum variations in OD after consecutive scans are calculated and compared to the values obtained for scans without interruption. For film irradiated to $3 \mathrm{~Gy}$, a time interval of 15 minutes between two successive scans leads to a reduction of the OD increase of $56.2 \%$ compared to when films are scanned without interruption. Reductions of OD increase of $86.72 \%$ and $78.72 \%$ respectively for film irradiated to $1 \mathrm{~Gy}$ and 2 Gy are obtained when a time interval of 30 minutes is left between two successive scans. These results show that when time intervals are observed between consecutive scans, the increase in OD has significantly reduced. However, the method has the drawback of increasing the time needed to perform radiochromic film dosimetry.
\end{abstract}

\section{Keywords}

Flatbed Scanner, Multiple Scans, Optical Density, Radiochromic Film

\section{Introduction}

Commonly available detectors used in medical applications give the possibility of obtaining the value of the absorbed dose at one point. Radiochromic films (RCF) offer the possibility of determining the absorbed dose over a large area. In 
addition, their tissue equivalence and near energy independence [1] [2] make them as preferable media for ionizing radiation dosimetry measurements like quality assurance [3] [4] [5] [6] and determination of dose distribution in radiotherapy [7] [8]. Another advantage is that they can be used in combination with a flatbed document scanner as densitometer [9] [10]. However, despite these advantageous properties if certain precautions are not taken, some artifacts may affect the accuracy of results obtained with radiochromic film dosimetry in combination with a flatbed scanner [11] [12]. In particular, the optical density (OD) of the films increases when these films are scanned in a repetitive manner. Lynch et al. [13] studied this phenomenon on Gafchromic EBT films using a Macbeth TD932 spot densitometer, an Epson Expression 1680 CCD array scanner, and a Microtek Scan Maker i900 CCD array scanner. They attributed this variation of optical density to the increase of the temperature of the scanner. On the other hand, Paelinck et al. [12] studied the same phenomenon on Gafchromic EBT films using an Epson Pro 1680 Expression flatbed scanner and attributed the increase of the OD to the UV component of the light of the scanner lamp.

Considering these observations, it is recommended to limit the number of the scans of single piece of film. However, some experiments such as the determination of the correction matrix described by Paelinck et al. [12] and investigations on scanning orientation effects in RCF dosimetry [13] [14] [15] require that a film is scanned several times. Therefore, it is important to develop strategies to minimize the variation of OD of RCFs in multiple scanning.

In this work, we investigate the effect of introducing a time gap between consecutives scans in order to reduce the increase in OD noticed in the literature in the special cases where multiple scanning of radiochromic film is required.

\section{Materials and Methods}

\subsection{Materials}

All experiments were carried out using Gafchromic EBT3 (International Specialty Products Corporation, Wayne, NJ, USA) lot no. 04061602 is used. Pieces of film $5 \times 5 \mathrm{~cm}^{2}$ were cut with sharp scissors from a sheet of $8 \times 10$. The Epson Pro 1680 Expression (Seiko Epson Corporation, Nagano, Japan) flatbed scanner was used as densitometer for scanning. Since the ambient temperature may influence the scanner response, the room temperature was stabilized using conditioned air at $26^{\circ} \mathrm{C}$. To avoid the artifacts associated with the presence of fingerprints, gloves were used during the manipulation of the films and the pieces of film were held on the edges. Films were handled in accordance with the recommendation outlined in AAPM TG-55 [1]. Generally, the films were removed from their light-protecting envelope only during cutting, irradiation and readout to reduce the effects of ambient light [16].

\subsection{Irradiation Procedure}

Once the films were cut, they were irradiated to doses of $1 \mathrm{~Gy}, 2 \mathrm{~Gy}$ or $3 \mathrm{~Gy}$. 
During the irradiation, each piece of film is placed in the central part of $10 \times 10$ $\mathrm{cm}^{2}$ field in a polystyrene (Polystyrol 495F, BASF, Germany) phantom at a depth of $10 \mathrm{~cm}$ and a SSD of $70 \mathrm{~cm}$. Additionally $10 \mathrm{~cm}$ of polystyrene was positioned under the film to ensure full backscatter conditions. Reference dosimetry was performed using a Farmer-type ionization chamber. All pieces of film were irradiated using an Alcyon II (G.E Medical System, USA) cobalt-therapy device (photon beam obtained from the decay of cobalt 60 of mean energy $1.25 \mathrm{MeV}$ ).

\subsection{Scanning Protocol}

The films were scanned at least forty-eight hours to ensure completion of the polymerization processes that leads to the radiation dependent color change in the gafchromic film [5] [17] [18]. The scanner was turned on thirty minutes before the beginning of each experiment. A single piece of film was scanned per day. The scans were performed using the Epson Scan software used in professional mode with all the options of image adjustments and color corrections disabled. The resolution was set to $72 \mathrm{dpi}$ (dot per inch), the scanner was used in transmission mode and remained closed throughout the experiment.

All pieces of film were scanned in the central area of the scanner using a homemade positioning device to ensure the reproducibility of the position of each piece of film to be scanned. All films were scanned in portrait orientation (original long side of the film parallel to the long side of the scanner bed, markers were placed on the square pieces of film to keep track of this orientation) and scanned on 48 bits in RGB color mode. The data are saved in the tagged image file format (TIFF). Paelinck et al. [13] in their study of Gafchromic EBT film with an EPSON 1680 Expression scanner showed that the optical density from the first scans are unreliable due to warm up of the lamp. Therefore, for all scan numbers in this work, the films were scanned five times and the OD was evaluated as the average OD of the last three scans. The region of interest was the 2 $\times 2 \mathrm{~cm}^{2}$ central part of the film (corresponding to $56 \times 56$ pixels ${ }^{2}$ ). Only the red channel was extracted from the original RGB file. The calculation of the optical density and the graphical representations are done using in-house written routines in the Matlab environment (The Math Works, Inc., Natwick, MA, USA, Matlab 7.9). Measured transmitted values were converted into raw $O D$ according to:

$$
O D=-\log \frac{I}{I_{0}}=\log \frac{P V_{0}}{P V}
$$

where $I_{0}$ and $I$ are the incident and transmitted light intensities, respectively. $P V_{0}$ and $P V$ are the corresponding pixels value.

\subsection{Temperature Effect during Multiple Scans}

In order to study multiple scanning effects Radiochromic film OD, pieces of EBT3 film were irradiated to $1 \mathrm{~Gy}, 2 \mathrm{~Gy}$ and $3 \mathrm{~Gy}$ then scanned forty times successively (a total of 200 scans considering the scanning procedure described 
above). Assuming that the increase in OD during a repetitive scan is mainly caused by an increase in temperature of the scanner lamp, a time interval was introduced between two consecutive scans is made in order to reduce temperature effects. Therefore, films irradiated to $1 \mathrm{~Gy}, 2 \mathrm{~Gy}$ and $3 \mathrm{~Gy}$ were scanned repetitively by maintaining the time intervals of fifteen minutes and thirty minutes between consecutive scans. The number of scans is reduced to twenty and fifteen scans respectively for time intervals of fifteen and thirty minutes between two consecutive scans. For the scan number $i$ of each series, the relative OD with respect to the first scan is calculated according to the following formula:

$$
\text { Relative change in } O D_{i}(\%)=\frac{\left[O D_{i}-O D_{1}\right]}{O D_{1}} \times 100
$$

\section{Results and Discussion}

The evolution of the relative change in OD obtained from forty consecutive scans without interruption of different pieces of film irradiated to $1 \mathrm{~Gy}, 2 \mathrm{~Gy}$ and $3 \mathrm{~Gy}$ is shown in Figure 1. The initial ODs of films irradiated to $1 \mathrm{~Gy} 2 \mathrm{~Gy}$ and 3 Gy were respectively $0.3020,0.3755$ and 0.4372 . A time period of about $4 \mathrm{~h}$ is needed to perform the 40 scans. Maximal values of relative change in OD observed are, $5.80 \%, 4.70 \%$ and $2.74 \%$ respectively for pieces of film irradiated to 1Gy, 2 Gy and 3 Gy. As already noticed by Lynch et al. (2006) and Paelinck et al. (2007), the increase of OD with repetitive scanning decreases as dose increases. A stabilization of the OD is noticed after scan number 15 for films irradiated to 1 Gy and scan 20 for films irradiated to 2 Gy and 3 Gy. This can be explained by fact that after an initial growth, the temperature of the scanner bed stabilizes.

Figure 2 presents the relative change in OD evolution for consecutive scans with interruptions of fifteen minutes between two scans for films irradiated to 1 Gy, 2 Gy and $3 \mathrm{~Gy}$. In order to reduce the time needed to perform the experiment, the number of scans is limited to 20. Maximum variations of relative change in OD of $2.41 \%, 1.67 \%$ and $1.20 \%$ are observed for pieces of film irradiated to $1 \mathrm{~Gy}, 2$ Gy and 3 Gy respectively. These values are lower than those obtained when the films are consecutively scanned without interruption. Compared to the consecutives scans without interruption, reduction in OD increase of $58.55 \%, 64.46 \%$ and $56.2 \%$ are obtained respectively for film irradiated to 1 Gy, 2 Gy and 3 Gy. Thus, an interruption of fifteen minutes between two consecutive scans significantly attenuates the OD variations.

When an interruption of thirty minutes is observed between two consecutive scans, the evolution of the relative change in OD is shown in Figure 3. In order to further reduce the time needed to perform the experiment, the number of scans is limited to fifteen. Maximal variations of OD of $0.77 \%, 1.00 \%$ and $1.16 \%$ are obtained respectively for pieces of film irradiated to $1 \mathrm{~Gy}, 2 \mathrm{~Gy}$ and $3 \mathrm{~Gy}$. After an initial increasing phase of the OD, a strong decreasing trend for pieces of film irradiated to 2 Gy and 3 Gy is observed after the fifth scan. Unlike the 


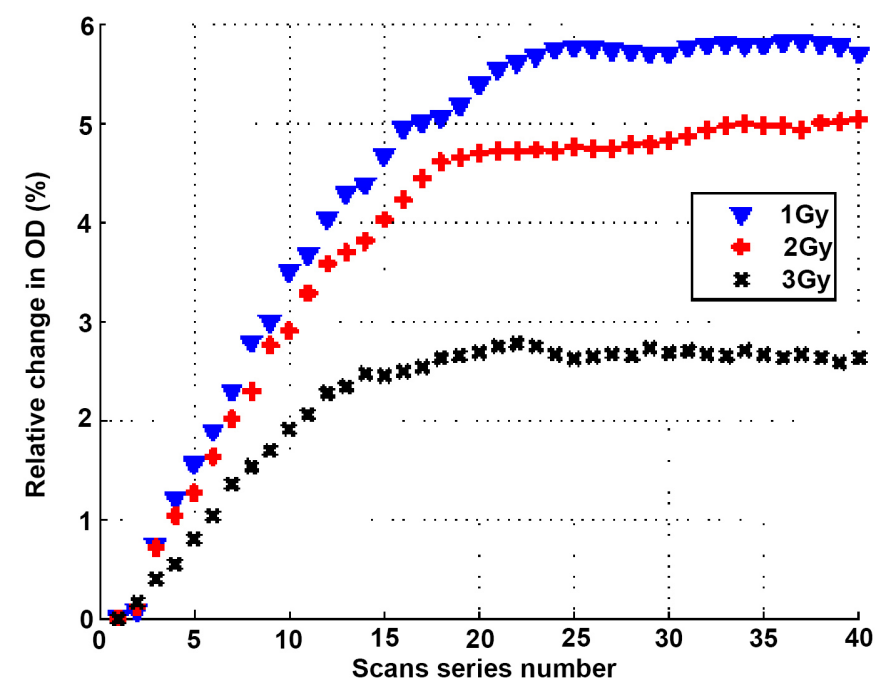

Figure 1. Evolution of the relative change in OD for pieces of film irradiated to $1 \mathrm{~Gy}, 2$ Gy and 3 Gy during consecutive scans without interruption.

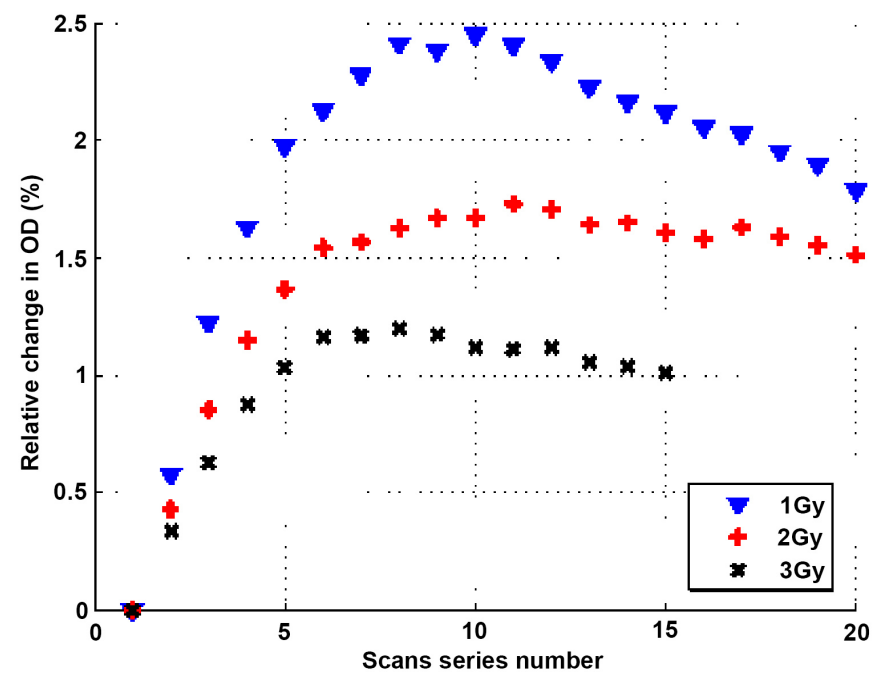

Figure 2. Evolution of the relative change in OD for pieces of film irradiated to $1 \mathrm{~Gy}, 2$ Gy and 3 Gy during consecutive scans with interruption of 15 minutes between two consecutive scans. Note Yscale on relative change in OD in this figure is different than previous figure.

cases where the films are scanned without interruption or with a break of fifteen minutes between two scans, the increase in OD with scan number is lower for higher doses. This can be explained by the fact that temperature effects being higher for lower doses, and any attempt to reduce temperature effects is therefore more efficient for low doses.

Reductions of the maximum variation of $86.72 \%, 78.72 \%$ and $57.66 \%$ respectively for films irradiated to $1 \mathrm{~Gy}, 2 \mathrm{~Gy}$ and $3 \mathrm{~Gy}$ are obtained in the case an interruption of thirty minutes is made between to scans compared to the case films are scanned without interruption. While increasing the time interval between 


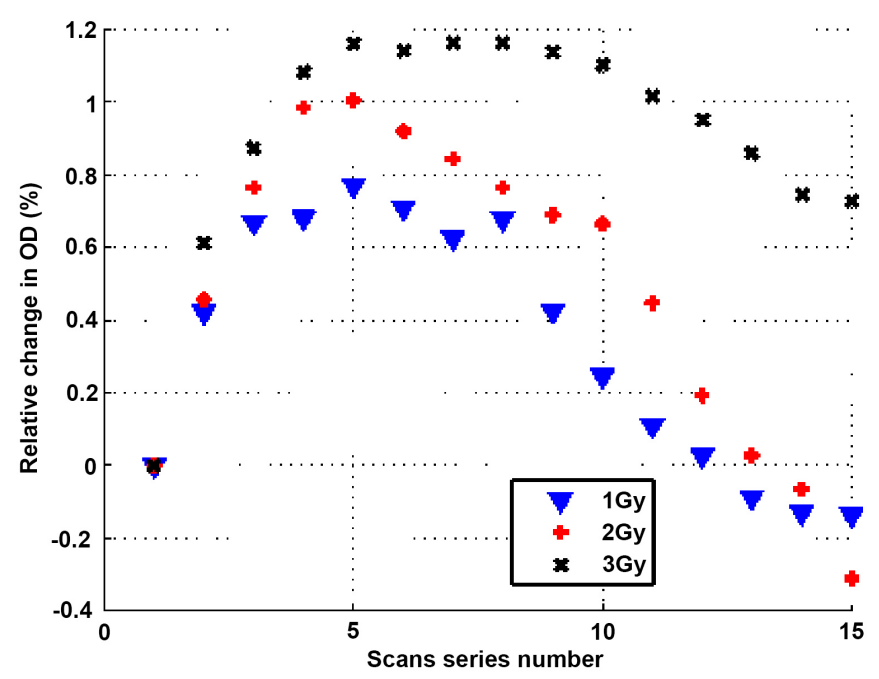

Figure 3. Evolution of the relative change in OD for pieces of film irradiated to $1 \mathrm{~Gy}, 2 \mathrm{~Gy}$ and $3 \mathrm{~Gy}$ during consecutive scans with interruption of 30 minutes between two consecutive scans. Note Yscale on relative delta OD in this figure is different than previous figures.

consecutives scans from fifteen to thirty minutes improves the efficiency of the reduction of the OD increase for films irradiated to $1 \mathrm{~Gy}$ and $2 \mathrm{~Gy}$, no significant amelioration was found for films irradiated to $3 \mathrm{~Gy}$. Therefore, for films irradiated to $3 \mathrm{~Gy}$, a time interval of fifteen minutes between consecutives scans sufficient in order to reduce the increase in OD caused by multiple scans of Radiochromic films.

For piece of film irradiated to $1 \mathrm{~Gy}$, a reduction of the maximum variation of $3.39 \%$ obtained in the case an interruption of 15 minutes is made between to scans compared to the case films are scanned without interruption. This value rises to $5.03 \%$ when the time interval between two scans is thirty minutes. For pieces of film irradiated to $2 \mathrm{~Gy}$, reduction of maximum variation in OD of $3.03 \%$ and $3.70 \%$ respectively for a times interval of 15 minutes and 30 minutes between two consecutives scans. These values drop to $1.54 \%$ and $1.58 \%$ for pieces of film irradiated to $3 \mathrm{~Gy}$ thus showing that the effect of increasing the time interval between two scans is less efficient for high doses.

\section{Conclusion}

This work shows that the increase in OD induced by multiple scanning of radiochromic film can be greatly reduced by maintaining time intervals between successive scans. For film irradiated to $3 \mathrm{~Gy}$, a time interval of 15 minutes between two successive scan leads to a reduction of the OD increase of $56.2 \%$ compared to when films are scanned without interruption. Reduction of OD increase of $86.72 \%$ and $78.72 \%$ respectively for film irradiated to 1 Gy and 2 Gy is obtained when a time interval of 30 minutes is left between two successive scans. However, this method has the drawback of increasing the time needed to per- 
form radiochromic films dosimetry. However, the method has the advantage to increase the accuracy of evaluation of OD (therefore of dose) in particular situations encountered during which multiples scans of films may be needed.

\section{Acknowledgements}

This work is partially supported by the ICTP through the OEA-AC-71 project.

\section{Conflicts of Interest}

The authors declare no conflicts of interest regarding the publication of this paper.

\section{References}

[1] Niroomand-Rad, A., Blackwell, C.R., Coursey, B.M., Gall, K.P., Galvin, J.M., McLaughlin, W.L., Meigooni, A.S., Nath, R., Rodgers, J.E. and Soares, C.G. (1998) Radiochromic Film Dosimetry: Recommandations of AAPM Radiation Therapy Committee Task Group 55. Medical Physics, 25, 2093-2115. https://doi.org/10.1118/1.598407

[2] Richter, C., Pawelke, J., Karsch, L. and Woithe, J. (2006) Energy Dependence of EBT-1 Radiochromic Film Response for Photon (10 kVp-15 MVp) and Electron Beams (6-18 MeV) Readout by a Flatbed Scanner. Medical Physics, 36, 5506-5514.

[3] Shi, C., Papanikolaou, N., Yan, Y., Weng, X. and Jiang, H. (2006) Analysis of the Sources of Uncertainty for EDR2 Film-Based IMRT Quality Assurance. Journal of Applied Clinical Medical Physics, 7, 1-8. https://doi.org/10.1120/jacmp.v7i2.2230

[4] Fuss, M., Sturtewagen, E., De Wagter, C. and Georg, D. (2007) Dosimetric Characterization of Gafchromic EBT Film and Its Implication on Film Dosimetry Quality Assurance. Physics in Medicine \& Biology, 52, 4211-4225.

https://doi.org/10.1088/0031-9155/52/14/013

[5] Soares, C.G., Trichter, S. and Davis, S.D. (2009) Radiochromic Film. In: Rogers, D.W.O. and Cygler, J.E., Eds., Summer School Clinical Dosimetry Measurements in Radiotherapy, AAPM Monograph, Medical Physics, Madison, WI, 759-813.

[6] Aland, T., Kairn, T. and Kenny, J. (2011) Evaluation of a Gafchromic EBT2 Film Dosimetry System for Radiotherapy Quality Assurance. Australasian Physical \& Engineering Sciences in Medicine, 34, 251-260. https://doi.org/10.1007/s13246-011-0072-6

[7] Fraass, B., Doppke, K., Hunt, M., Kutcher, G., Starkschall, G., Stern, R. and Van Dyke, J. (1998) American Association of Physicists in Medicine Radiation Therapy Committee Task Group 53: Quality Assurance for Clinical Radiotherapy Treatment Planning. Medical Physics, 25, 1773-1829. https://doi.org/10.1118/1.598373

[8] Sankar, A., Goplakrishna Kurup, P.G., Muralli, V., Ayyangar, K.M., Mothilal Nahru, R. and Velmurugan, J. (2006) Evaluation of Gafchromic EBT Film for Intensity Modulated Radiation Therapy Dose Distribution Verification. Medical Physics, 31, 78-82. https://doi.org/10.4103/0971-6203.26693

[9] Williams, M. and Metcalfe, P.E. (2011) Radiochromic Film Dosimetry and Its Applications in Radiotherapy. AIP Conference Proceedings, 1345, 75-99.

https://doi.org/10.1063/1.3576160

[10] Matney, J.E., Parker, B.C., Neck, D.W., Henkelmann, G. and Rosen, I.I. (2010) Evaluation of Commercial Flatbed Document Scanner and Radiographic Film 
Scanner for Radiochromic EBT Film Dosimetry. Journal of Applied Clinical Medical Physics, 11, 198-208. https://doi.org/10.1120/jacmp.v11i2.3165

[11] Stevens, M.A., Turner, J.R., Hugtenburg, R.P. and Butler, P.H. (1996) High-Resolution Dosimetry Using Radiochromic Film and a Document Scanner. Physics in Medicine \& Biology, 41, 2357-2365. https://doi.org/10.1088/0031-9155/41/11/008

[12] Alnawaf, H., Yu, P.K.N. and Butson, M.J. (2012) Comparison of Epson Scanner Quality for Radiochromic Film Evaluation. Journal of Applied Clinical Medical Physics, 13, 314-321. https://doi.org/10.1120/jacmp.v13i5.3957

[13] Paelinck, L., De Neve, W. and De Wagter, C. (2007) Precautions and Strategies in Using a Commercial Flatbed Scanner for Radiochromic Film Dodimétry. Physics in Medicine \& Biology, 52, 231-242. https://doi.org/10.1088/0031-9155/52/1/015

[14] Lynch, B.D., Kazelka, J., Kanade, M.K., Li, J.G., Simon, W.E. and Dempsey, J.F. (2006) Important Considerations for Radiochromic Film Dosimetry with Flatbed CCD Scanners and EBT Gafchromic Film. Medical Physics, 33, 4551-4556. https://doi.org/10.1118/1.2370505

[15] Butson, M.J., Yu, P.K.N., Cheung, T. and Inwood, D. (2003) Polarization Effects on a High-Sensitivity Radiochromic Film. Physics in Medicine \& Biology, 48, N207-N211. https://doi.org/10.1088/0031-9155/48/15/401

[16] Butson, M.J., Cheung, T. and Yu, P.K.N. (2006) Scanning Orientation Effect on Gafchromic EBT Film Dosimetry. Australasian Physical and Engineering Sciences in Medicine, 29, 281-284. https://doi.org/10.1007/BF03178579

[17] Butson, M.J., Yu, P.K.N. and Metcalfe, P.E. (1998) Effects of Read-Out Light Sources and Ambient Light on Radiochromic Film. Physics in Medicine \& Biology, 43, 2407-2412. https://doi.org/10.1088/0031-9155/43/8/031

[18] Cheung, T., Butson, M.J. and Yu, P.K.N. (2005) Post-Irradiation Colouration of Gafchromic EBT Radiochromic Film. Physics in Medicine \& Biology, 50, N281-N285.

https://doi.org/10.1088/0031-9155/50/20/N04 